\title{
Investigation of controlled external feedback on the properties of low and high-power frequency-stabilized diode laser
}

Zink, Christof; Christensen, Mathias; Jamal, Muhammad Tahir; Hansen, Anders Kragh; Maiwald, Martin; Jensen, Ole Bjarlin; Sumpf, Bernd ; Tränkle, Günther

\section{Published in:}

Proceedings of SPIE

Link to article, DOI:

$10.1117 / 12.2509546$

Publication date:

2019

Document Version

Publisher's PDF, also known as Version of record

Link back to DTU Orbit

Citation (APA):

Zink, C., Christensen, M., Jamal, M. T., Hansen, A. K., Maiwald, M., Jensen, O. B., Sumpf, B., \& Tränkle, G. (2019). Investigation of controlled external feedback on the properties of low and high-power frequencystabilized diode laser. In Proceedings of SPIE (Vol. 10939). [109391J] SPIE - International Society for Optical Engineering. Proceedings of SPIE - The International Society for Optical Engineering https://doi.org/10.1117/12.2509546

\section{General rights}

Copyright and moral rights for the publications made accessible in the public portal are retained by the authors and/or other copyright owners and it is a condition of accessing publications that users recognise and abide by the legal requirements associated with these rights.

- Users may download and print one copy of any publication from the public portal for the purpose of private study or research.

- You may not further distribute the material or use it for any profit-making activity or commercial gain

- You may freely distribute the URL identifying the publication in the public portal 


\section{Investigation of controlled external feedback on the properties of low and high-power frequency-stabilized diode laser}

Christof Zink, Mathias Christensen, Muhammad T. Jamal, Anders K. Hansen, Martin Maiwald, et al.

Christof Zink, Mathias Christensen, Muhammad T. Jamal, Anders K. Hansen, Martin Maiwald, Ole B. Jensen, Bernd Sumpf, Günther Tränkle,

"Investigation of controlled external feedback on the properties of low and high-power frequency-stabilized diode laser," Proc. SPIE 10939, Novel InPlane Semiconductor Lasers XVIII, 109391J (1 March 2019); doi: $10.1117 / 12.2509546$

SPIE. Event: SPIE OPTO, 2019, San Francisco, California, United States 


\title{
Investigation of controlled external feedback on the properties of low and high-power frequency stabilized diode lasers
}

\author{
Christof Zink ${ }^{1}$, Mathias Christensen ${ }^{2}$, Muhammad T. Jamal ${ }^{2}$, Anders K. Hansen², Martin Maiwald ${ }^{1}$, \\ Ole B. Jensen ${ }^{2}$, Bernd Sumpf ${ }^{1}$, and Günther Tränkle ${ }^{1}$ \\ ${ }^{1}$ Ferdinand-Braun-Institut, Leibniz-Institut für Höchstfrequenztechnik, Gustav-Kirchhoff-Str. 4, \\ 12489 Berlin \\ ${ }^{2}$ DTU Fotonik, Technical University of Denmark, Frederiksborgvej 399, 4000 Roskilde, Denmark
}

\begin{abstract}
Unwanted optical feedback is a common problem in many optical setups of laser systems. To quantify the effect, the influence of a controlled external feedback on the emission properties of a low power distributed feedback ridge waveguide and a high power distributed Bragg reflector tapered diode laser are analyzed. The measured influence of the phase dependent feedback over several orders of magnitude in feedback power attenuation on emission wavelength is discussed and compared to theory.
\end{abstract}

Keywords: Diode laser, distributed Bragg reflector, feedback, tapered diode laser

\section{INTRODUCTION}

The advances in diode laser technology enable a growing number of scientific experiments and commercial applications. A common problem for many experiments and applications is unwanted optical feedback from the setup itself to the diode laser. Each optical surface in the beam path of the laser generates either a reflected beam or a scatter light field. Unwanted feedback is introduced, if parts of the reflected beam or the scatter light reach the diode laser. The effects of optical feedback on the laser emission can vary from emission frequency shifts, small power alterations and changes in the spectral line width for weak feedback, but it can also lead to coherence collapse or even device damage for higher feedback powers. These effects for weak external feedback were so far analyzed for low power diode lasers with an additional frequency selective element like distributed feedback (DFB) or distributed Bragg reflector (DBR) lasers. For high power lasers the introduced changes to the laser emission by weak external feedback were typically small compared to the variance of the laser emission parameters without feedback. However, the development of high power frequency stabilized diode lasers with an almost diffraction limited beam quality factor as well as narrowband and stable spectral emission, and new and increasingly demanding applications like efficient frequency doubling require an investigation on the effects of controlled weak external feedback on high power diode lasers.

In this paper we present the results of the investigation of controlled weak external feedback on two types of diode lasers. A low power $(140 \mathrm{~mW})$ DFB laser is used to qualify the setup and as a reference and comparison to already published results. A DBR tapered diode laser is used as an example of a high power $(2 \mathrm{~W})$ diode laser. The influence of small changes in the length of the external feedback cavity on the emission frequency is compared for several feedback power ratios with simulations.

\section{THEORY}

The common approach to analyze the effects of external feedback on the emission characteristics of a diode lasers ${ }^{1,2}$ uses the field equation of lasers with an additional source term in form of time delayed reinjection of the field, the so called Lang Kobayashi equation ${ }^{3}$. This equation is an approximation for a Fabry Perot type laser without additional frequency selective elements and without noise terms.

$$
\frac{d}{d t} E(t) e^{-i \omega t}=\left(-i \omega_{0}+\frac{1}{2} \Delta G(1-i \alpha)\right) E e^{-i \omega t}(t)+\kappa E\left(t-\tau_{e}\right) e^{-i \omega t}
$$

Novel In-Plane Semiconductor Lasers XVIII, edited by Alexey A. Belyanin,

Peter M. Smowton, Proc. of SPIE Vol. 10939,109391J · C) 2019 SPIE

CCC code: $0277-786 X / 19 / \$ 18 \cdot$ doi: $10.1117 / 12.2509546$ 
where $\omega_{0}$ is the emission frequency of the laser without feedback and $\omega$ is the emission frequency with external feedback. $\Delta G$ is the introduced gain change by external feedback and $\alpha$ the linewidth enhancement factor. The coefficient $\kappa$ is related to the cavity parameters of the setup:

$$
\kappa=\frac{\left(1-R_{s}\right) \sqrt{R_{e}}}{\tau_{s} \sqrt{R_{s}}}
$$

with $R_{S}$ the effective front facet reflectivity of the diode laser, $R_{\mathrm{e}}=10^{\left(\mu_{A T} / 10\right)}$ the reflectivity of the external feedback including coupling losses and attenuation $\mu_{A T}$. The internal round-trip time in the diode laser is given by $\tau_{S}$ and the round-trip time of the external resonator by $\tau_{e}$.

The frequency shift due to external feedback can be approximated by

$$
\Delta \omega=\omega-\omega_{0}=-\kappa\left(\sin \left(\omega \tau_{e}\right)+\alpha \cos \left(\omega \tau_{e}\right)\right)
$$

and the maximum frequency shift by

$$
\Delta \omega_{\max }=2 \pi \Delta v_{\max }=-\kappa \sqrt{1+\alpha^{2}}
$$

The frequency shift in dependence of the feedback mirror displacement and therefore the introduced change in the external round-trip time $\tau_{e}$ is plotted in Fig. 1. It is plotted for 4 different values of $\kappa \tau_{e}$, with increasing feedback from top to bottom. The top two graphs of Fig. 1 show the simulations for very weak external feedback. At these feedback levels the frequency shifts continuously with the feedback mirror displacement and the maximum frequency shift increases with the amount of feedback $\kappa \tau_{e}$. In the third graph from top the waveform of the frequency shift has regions with multiple solutions highlighted in grey for the same external cavity round-trip time $\tau_{e}$. This leads to a hysteresis based on the change in $\tau_{e}$ or the movement direction of the external feedback mirror. The arrows and the color indicate the direction of the mirror movement: green towards the diode laser and blue away from the diode laser. For the lowest graph 3 characteristic values of the frequency shift waveform are highlighted in orange, they are: maximum frequency shift $\Delta v_{\max }$, number of maximal simultaneous possible modes $m_{\max }$, and the mode spacing between neighboring modes $\Delta v_{\text {mod }}$.

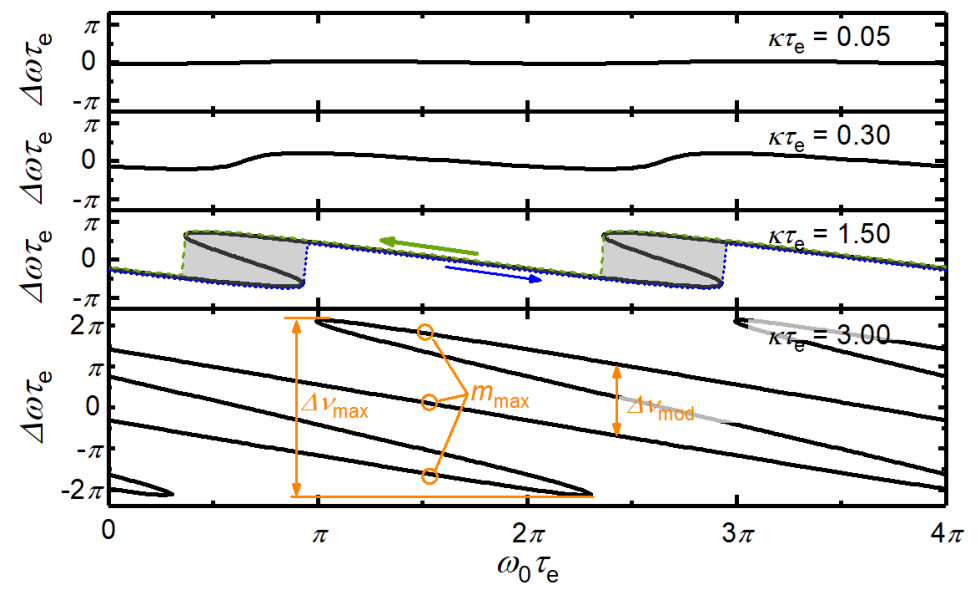

Fig. 1 Simulated frequency shift in dependence of the feedback mirror displacement for 4 different amounts of feedback powers $k \tau_{\mathrm{e}}$. The dependence of the frequency shift on the mirror movement direction and the resulting hysteresis is shown for $\kappa \tau_{\mathrm{e}}=1.50$. Three characteristic values can be read of the frequency shift curve: the maximum frequency shift $\Delta v_{\max }$, the maximum number of parallel possible modes $m_{\max }$ and the mode spacing between neighboring modes $\Delta v_{\bmod }$.

\section{EXPERIMENTAL RESULTS}

\subsection{Distributed feedback diode laser}

The DFB laser shown as a sketch in Fig. 1 was produced at the FBH and is used in this investigation as a reference laser to test the setup. Its vertical layer structure is based on an InGaAs single quantum well embedded in a $3.6 \mu \mathrm{m}$ wide super 
large optical cavity. The first order Bragg grating with a design wavelength of $975 \mathrm{~nm}$ was formed in a 2 step process by holographic photolithography and wet-chemical etching ${ }^{4}$. The device has a length of $L_{\mathrm{DFB}}=1.5 \mathrm{~mm}$ and lateral confinement is provided by a $w_{\mathrm{RW}}=3 \mu \mathrm{m}$ wide dry etched ridge waveguide (RW). A highly reflective coating with a design reflectivity of $R_{\mathrm{HR}}=95 \%$ was applied to the back facet while an anti-reflection coating with $R_{\mathrm{AR}}<0.1 \%$ was applied to the front facet.

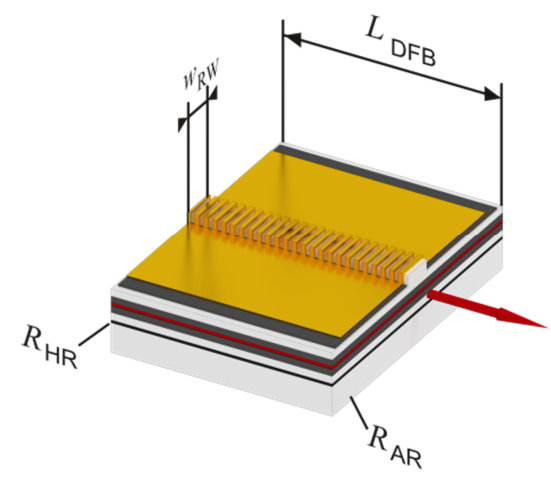

Fig. 2 Sketch of the DFB laser

The power voltage current characteristic is show in Fig. 3 a) with the optical output power in blue, the voltage in black and the calculated electro-optical efficiency in red. The injection current was increased from 0 to $500 \mathrm{~mA}$ with a step size of $2 \mathrm{~mA}$. The device starts lasing at a threshold current of $I_{\mathrm{DFB}}$, thr $=25 \mathrm{~mA}$ and the output power increases with the injection current with a slope of $0.8 \mathrm{~W} / \mathrm{A}$. The characteristic line is kink free over the full measurement range and a maximum output power of $P=350 \mathrm{~mW}$ at $I_{\mathrm{DFB}}=500 \mathrm{~mA}$ was measured. The highest electro-optical efficiency of $32 \%$ was measured at $I_{\mathrm{DFB}}=156 \mathrm{~mA}$. In addition to the output power and the voltage a spectrum was measured for each injection current. These spectra were individually normalized and are shown as a false color map plot in Fig. 3 b). At threshold the laser emits at $\lambda=974.0 \mathrm{~nm}$ and with increasing injection current the wavelength shifts towards longer wavelength resulting in an emission wavelength of $\lambda=975.7 \mathrm{~nm}$ at $I_{\mathrm{DFB}}=500 \mathrm{~mA}$. Over the whole measured current range the emission is spectrally single mode with a measured spectral width (FWHM) $\Delta \lambda=20 \mathrm{pm}$, limited by the resolution of the spectrometer. An injection current of $I_{\mathrm{DFB}}=200 \mathrm{~mA}$ was chosen for the following feedback measurements. At this injection current the laser emits $140 \mathrm{~mW}$ of optical output power with spectrum shown in Fig. $3 \mathrm{c})$.
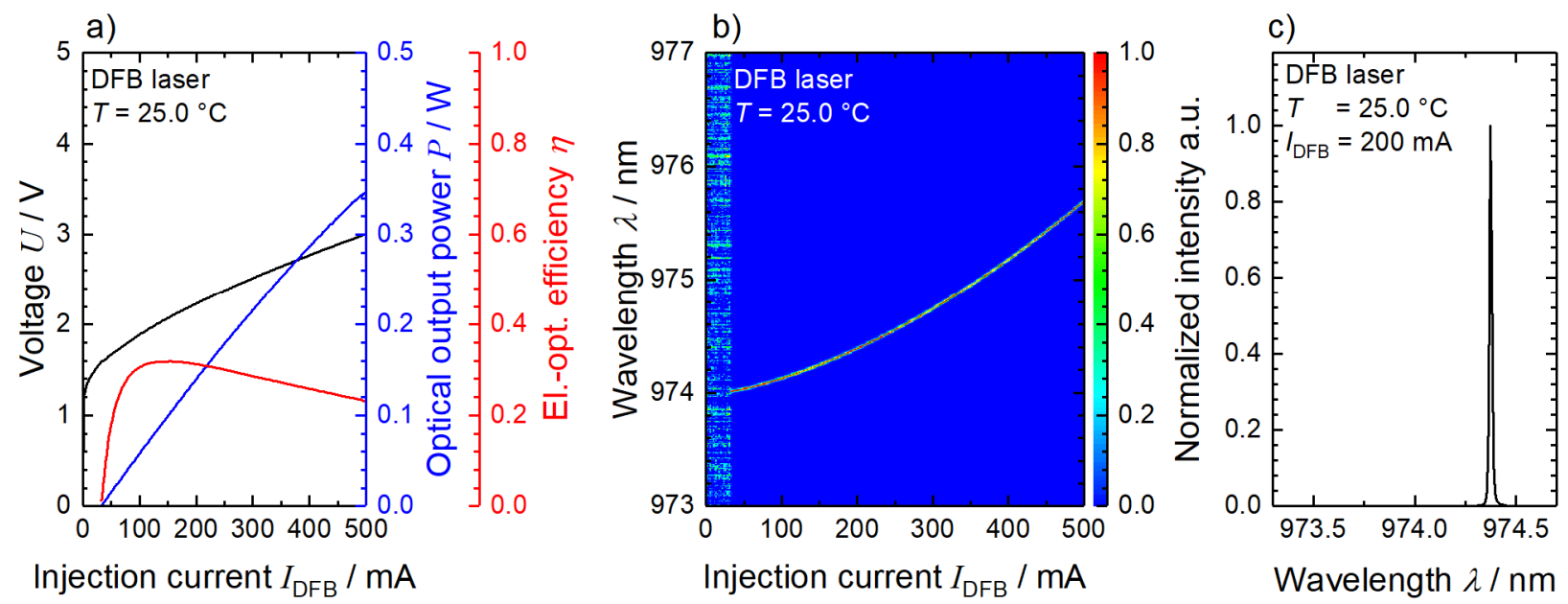

Fig. 3 a) Power voltage current characteristic and the electro-optical efficiency of the DFB laser. b) False color map plot of the emission spectra over the injection current $I_{\mathrm{DFB}}$. c) Single mode emission spectrum at $I_{\mathrm{DFB}}=200 \mathrm{~mA}$. 
Fig. 4 a) shows a simplified sketch of the setup for the investigation on the effects of controlled external feedback on the emission parameters of a diode laser. The laser is mounted on a Peltier controlled heat sink. The emitted beam is collimated with a biconvex anti-reflection coated aspherical lens $(f=2 \mathrm{~mm})$. For the DBR tapered laser an additional anti-reflection coated cylindrical lens $(f=5.8 \mathrm{~mm})$ is used to compensate the astigmatism. A portion of the collimated beam is picked up by an uncoated wedge for the feedback branch. A variable reflective filter is used to control the power of the beam before it is focused with a $f=45 \mathrm{~mm}$ anti-reflection coated lens on the feedback mirror. The feedback mirror is placed in a distance of $L_{\mathrm{FM}}=350 \mathrm{~mm}$ to the front facet of the laser and is mounted on a piezo actuator. With the piezo actuator the feedback mirror can be displaced along the beam path changing distance $L_{\mathrm{FM}}=350 \mathrm{~mm}$ by up to $2 \mu \mathrm{m}$. The introduced changes to the beam profile over the complete mirror displacement range are very small and can be neglected. The beam passing the wedge in transmission direction is then transmitted through a Faraday isolator onto a beam dump. A portion of the scattered light is collected with an optical fiber an then analyzed with a scanning Fabry Perot interferometer with a free spectral range of $v_{\mathrm{FSR}}=1.5 \mathrm{GHz}$ and a resolution of $\Delta v=67 \mathrm{MHz}$. The false color map plot of the interferogram shown in top graph of Fig. 4 b) was measured for the DFB laser and a feedback attenuation of $\mu_{\mathrm{AT}}=-50 \mathrm{~dB}$ by applying a triangular voltage waveform to the Piezo actuator of the feedback mirror. Below the interferogram the applied piezo voltage $U_{\mathrm{P}}$ (middle graph) and the resulting feedback mirror displacement $\Delta L_{\mathrm{FM}}$ (lower graph) is plotted. The blue symbols indicating a mirror movement direction away from the laser and the green symbols a mirror movement towards the laser. A more detailed description of the setup and the used measurement method is described elsewhere.

a)

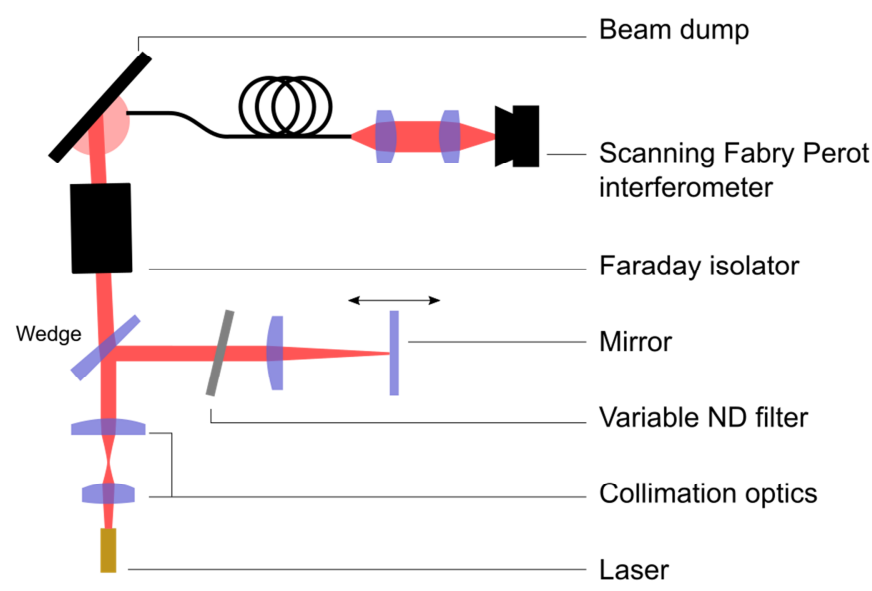

b)

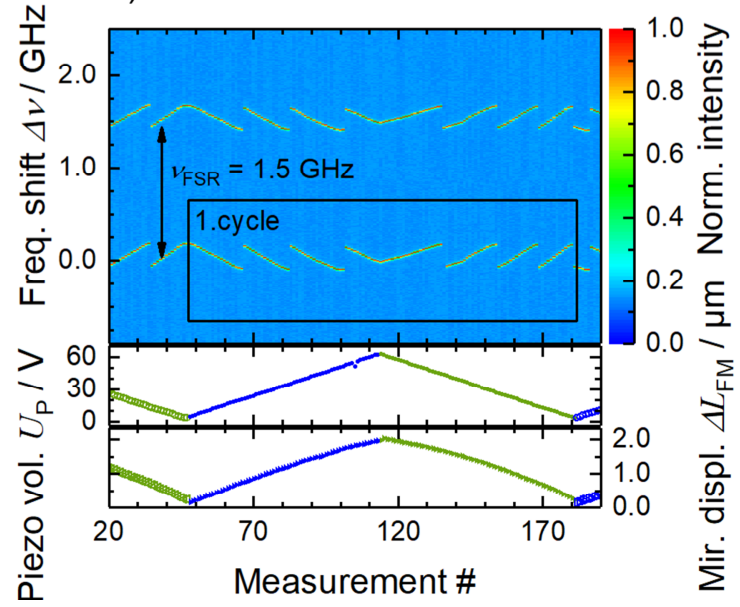

Fig. 4 a) Sketch of the feedback measurement setup. b) False color map plot of the spectra measured with the scanning Fabry Perot interferometer is shown on the top. A full cycle of the feedback mirror movement is highlighted by the black box. The two lower graphs show the applied piezo voltage and the resulting mirror displacement.

For the feedback measurements the feedback power attenuation was increased from $\mu_{\mathrm{AT}}=-90 \mathrm{~dB}$ to $\mu_{\mathrm{AT}}=-40 \mathrm{~dB}$ with a step size of $\Delta \mu_{\mathrm{AT}}=5 \mathrm{~dB}$. Fig. 5 a) shows exemplarily the frequency shift for the DFB laser diode in dependence of the feedback mirror displacement $\Delta L_{\mathrm{FM}}$ for 4 different feedback attenuations $\mu_{\mathrm{AT}}$. The color of the symbols shows the movement direction of the feedback mirror (blue: away from the diode laser and green: towards the diode laser). The solid black line shows the calculated frequency shift according to Eq. (1). At a feedback attenuation of $\mu_{\mathrm{AT}}=-90 \mathrm{~dB}$ the frequency shift was not resolvable. With increasing feedback power the maximum frequency shift increases and the frequency shifts continuously with the mirror displacement as shown for $\mu_{\mathrm{AT}}=-75 \mathrm{~dB}$ and $\mu_{\mathrm{AT}}=-60 \mathrm{~dB}$. If the feedback power is increased further the frequency shift becomes discontinuous and dependent on the movement direction of the feedback mirror as shown in the lowest graph of Fig. 5 a) for $\mu_{\mathrm{AT}}=-45 \mathrm{~dB}$. The maximum frequency shift $\Delta v_{\max }$, the maximum number of simultaneous possible modes $m_{\max }$ and the mode spacing between neighboring modes $\Delta v_{\max }$ were measured for each set of feedback power attenuation. The corresponding graphs are shown in Fig. $5 \mathrm{~b}$ )-d). The device length of $L_{\mathrm{DFB}}=1.5 \mathrm{~mm}$ and the external cavity with distance to the feedback mirror of $L_{\mathrm{FM}}=350 \mathrm{~mm}+\Delta L_{\mathrm{FM}}$ result in an internal round-trip time of $\tau_{\mathrm{s}}=34 \mathrm{ps}$ and an external round-trip time of $\tau_{\mathrm{e}}=2.3 \mathrm{~ns}$. The linewidth enhancement factor of $\alpha=2$ is a typical value for a DFB laser with this epitaxial structure and this emission wavelength. The reflectivity of the external feedback $R_{\mathrm{e}}$ was adjusted by the variable ND filter as described 
above. The effective front facet reflectivity of $R_{\mathrm{S}}=5 \%$ is the result of fitting eq. (2) to the measured data of the maximum frequency shift. This value is significantly higher than the specified anti-reflection coating $R_{\mathrm{AR}}<0.1 \%$, but due to the integrated DFB grating the effective front facet reflectivity may be higher than the reflectivity of the AR coating. Nevertheless, the simulated data based on the above-mentioned values is in excellent agreement with the measured data over the whole measured data range as can be seen in all graphs of Fig. 5. The experimental results and the good agreement with the simulation confirm that the measurement method and the setup can be used to characterize the influence of controlled external feedback on diode laser. In the next paragraph experimental results of the same measurement method are presented for the DBR tapered diode laser as an example for a high power diode laser.
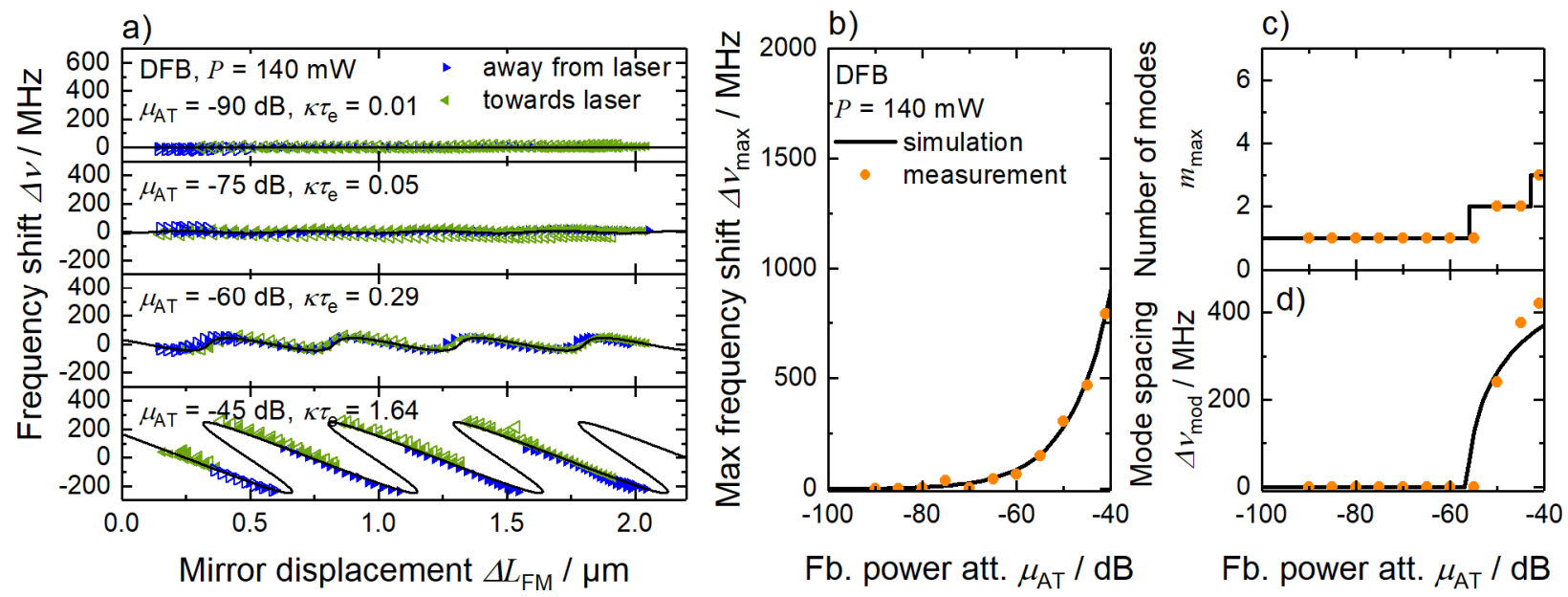

Fig. 5 a) Frequency shift over the mirror displacement for 4 different feedback power attenuations $\mu_{\mathrm{AT}}$ for the DFB diode laser. The mirror movement direction is shown by the color of the symbols (green: towards laser diode, blue: away from laser diode). b) The maximum frequency shift $\Delta v_{\max }, \mathrm{c}$ ) the maximum number of simultaneous possible modes and d) the mode spacing between neighboring modes are plotted over the feedback power attenuation $\mu_{\mathrm{AT}}$. The measured values (symbols) are compared for all graphs with simulation (solid black line).

\subsection{Distributed Bragg reflector tapered diode laser}

A sketch of the DBR tapered diode laser is shown in Fig. 6, it was manufactured at the FBH and is used to investigate the influence of feedback on a high power frequency stabilized diode laser. Its vertical layer structure is based on an InGaAs triple quantum well embedded in a $4.8 \mu \mathrm{m}$ wide asymmetrical super large optical cavity. The $7^{\text {th }}$ order Bragg grating with a design wavelength of $1066 \mathrm{~nm}$ was realized by electron-beam lithography and is etched partly through the p-side of the vertical layer structure. The device has a full length of $L_{\mathrm{DBR}-\mathrm{TPL}}=6.0 \mathrm{~mm}$ and consists of three sections. A $L_{\mathrm{DBR}}=1.0 \mathrm{~mm}$ long passive tapered DBR grating section, a $L_{\mathrm{RW}}=1.5 \mathrm{~mm}$ long active $\mathrm{RW}$ section and a $L_{\mathrm{TA}}=3.5 \mathrm{~mm}$ long tapered amplifier (TA) section. The lateral confinement for the RW section is provided by a $w_{\mathrm{RW}}=5 \mu \mathrm{m}$ wide dry etched ridge waveguide (RW). The shape of the TA section is defined by the p-side contact of this section. The full tapered angle for this device is $\alpha_{\mathrm{TA}}=6^{\circ}$. An anti-reflective coating $R_{\mathrm{AR}}<0.1 \%$. was applied to the rear facet and the front facet was coated to reduce the reflectivity to $R_{\mathrm{FF}}=0.3 \%$. 


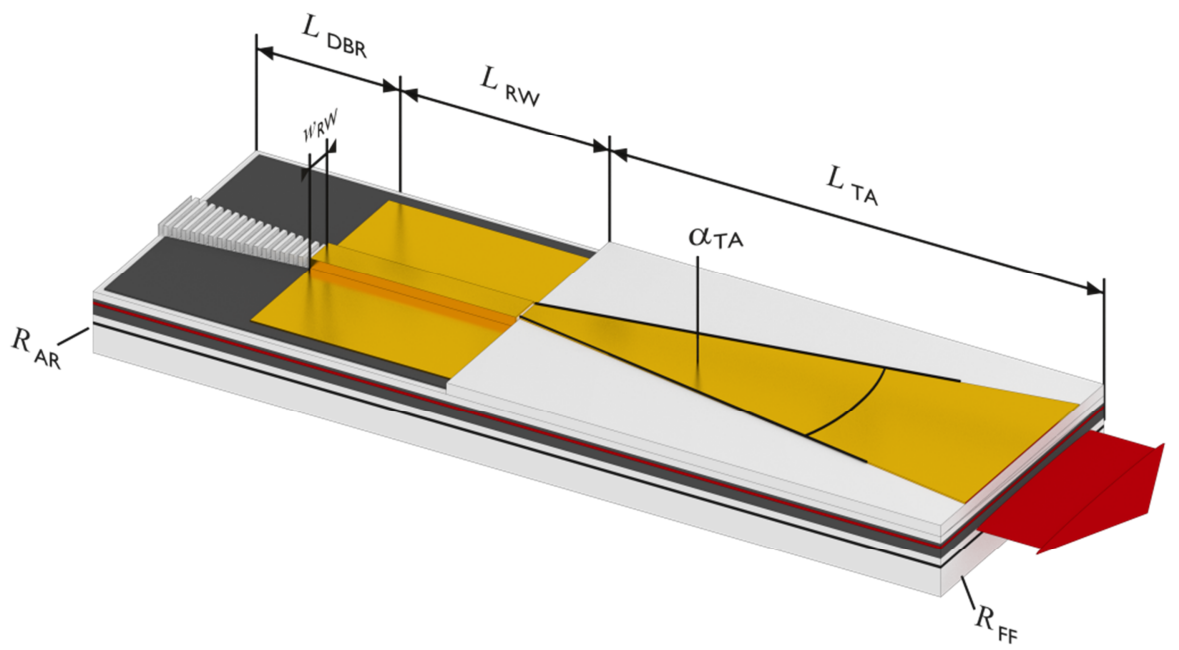

Fig. 6 Sketch of the DBR tapered diode laser

Fig. 7 a) shows the power voltage current characteristic line with the optical output power in blue, the voltage in black and the calculated electro optical efficiency in red for the DBR tapered diode laser. The injection current to the RW section was set to $I_{\mathrm{RW}}=300 \mathrm{~mA}$ and the injection current to the tapered section was increased from $I_{\mathrm{TA}}=0$ to $10 \mathrm{~A}$ with a step size of $0.1 \mathrm{~A}$. The device starts lasing at a threshold current of $I_{\mathrm{TA}, \text { thr }}=1.7 \mathrm{~A}$ and the output power increases with the injection current with a slope of 0.7 W/A. The characteristic is kink free over the full measurement range and a maximum output power of $P=5.7 \mathrm{~W}$ at $I_{\mathrm{TA}}=10 \mathrm{~A}$ was measured. The highest electro-optical efficiency of $32 \%$ was measured at $I_{\mathrm{TA}}=8.2 \mathrm{~A}$. In addition to the output power and the voltage a spectrum was measured for each injection current. These spectra were individually normalized and are shown as a false color map plot in Fig. 7 b). At threshold the laser emits at $\lambda=1065.8 \mathrm{~nm}$ and with increasing injection current the wavelength shifts towards longer wavelength with a slope of $\Delta \lambda=96 \mathrm{pm} / \mathrm{A}$. Small mode jumps $\Delta \lambda_{\mathrm{MOD}} \approx 28 \mathrm{pm}$ corresponding to the longitudinal modes of an internal resonator occur above threshold. Above $I_{\mathrm{TA}}=3 \mathrm{~A}$ the emission is spectrally single mode with a measured spectral width (FWHM) $\Delta \lambda=20 \mathrm{pm}$, limited by the resolution of the spectrometer. The injection currents of $I_{\mathrm{RW}}=300 \mathrm{~mA}$ and $I_{\mathrm{TA}}=5 \mathrm{~A}$ were chosen for the following feedback measurements. At these settings laser emits $2 \mathrm{~W}$ of optical output power with spectrum shown in Fig. 7 c).
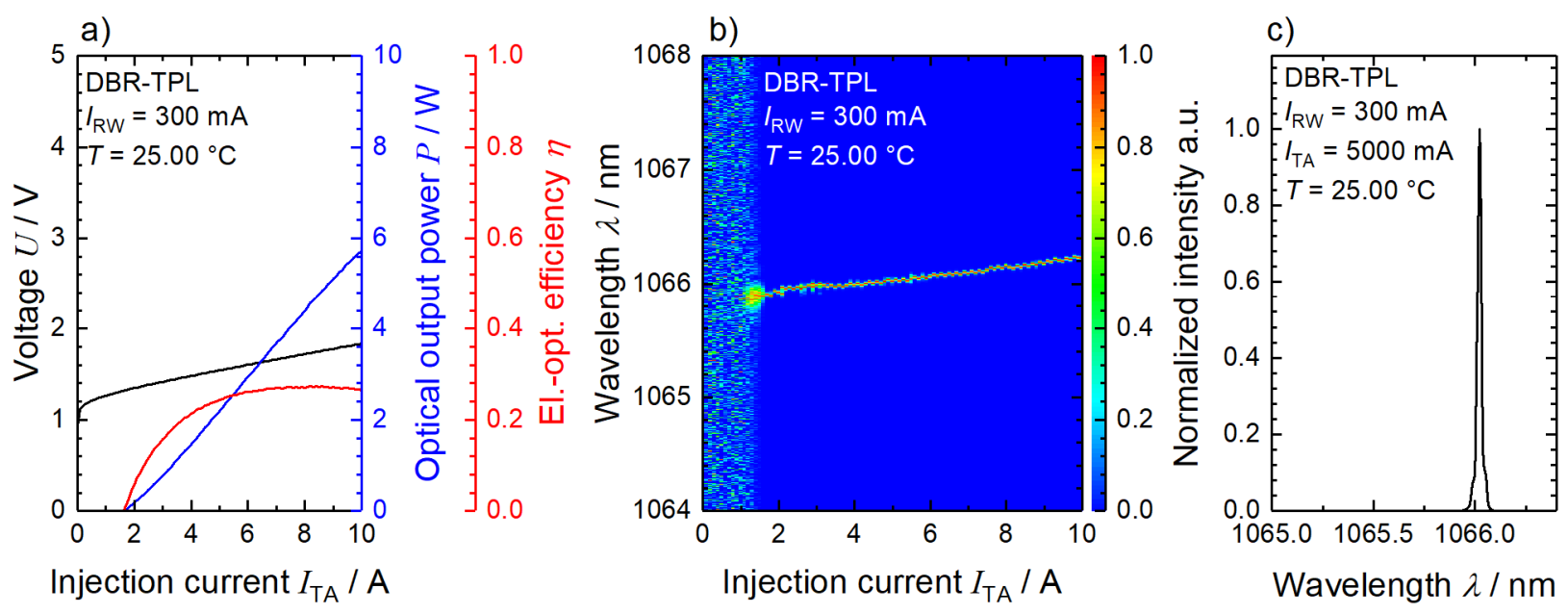

Fig. 7 a) Power voltage current characteristic and the electro-optical efficiency of the DBR-TPL for $I_{\mathrm{RW}}=300 \mathrm{~mA}$. b) False color map plot of the emission spectra over the injection current $I_{\mathrm{TA}}$. c) Single emission spectrum at $I_{\mathrm{RW}}=300 \mathrm{~mA}$ and $I_{\mathrm{TA}}=5000 \mathrm{~mA}$. 
The feedback measurements with the DBR tapered diode laser were performed with a feedback power attenuation from $\mu_{\mathrm{AT}}=-105 \mathrm{~dB}$ to $\mu_{\mathrm{AT}}=-40 \mathrm{~dB}$ with a step size of $\Delta \mu_{\mathrm{AT}}=5 \mathrm{~dB}$. Fig. $8 \mathrm{a}$ ) shows exemplarily the frequency shift for the laser diode in dependence of the feedback mirror displacement $\Delta L_{\mathrm{FM}}$ for 4 different feedback attenuations $\mu_{\mathrm{AT}}$. The color of the symbols show again the movement direction of the feedback mirror (blue: away from the diode laser and green: towards the diode laser) and the solid black line shows the calculated frequency shift according to Eq. (1). At a feedback attenuation of $\mu_{\mathrm{AT}}=-105 \mathrm{~dB}$ the frequency shift was not resolvable. With increasing feedback power the maximum frequency shift increases and the frequency shifts continuously with the mirror displacement as shown for $\mu_{\mathrm{AT}}=-85 \mathrm{~dB}$. At around $\mu_{\mathrm{AT}}=-80 \mathrm{~dB}$ the frequency shift becomes discontinuous and dependent on the movement direction of the feedback mirror as shown in third graph from the top of Fig. 8 a) for $\mu_{\mathrm{AT}}=-70 \mathrm{~dB}$. At a feedback power attenuation of $\mu_{\mathrm{AT}}=-45 \mathrm{~dB}$ the frequency shifts still discontinuously with the mirror displacement position but no movement direction dependent hysteresis was observed. The maximum frequency shift $\Delta v_{\max }$, the maximum number of simultaneous possible modes $m_{\max }$ and the mode spacing between neighboring modes $\Delta v_{\max }$ were measured for each set of feedback power attenuation. The corresponding graphs are shown in Fig. $8 \mathrm{~b}$ )-d). The device length of $L_{\mathrm{DBR}-\mathrm{TPL}}=6 \mathrm{~mm}$ gives an internal round-trip time of $\tau_{\mathrm{s}}=125 \mathrm{ps}$ and the external round-trip time is again $\tau_{\mathrm{e}}=2.3 \mathrm{~ns}$. The linewidth enhancement factor of $\alpha=2$ is a typical value for a DBR tapered diode laser $^{6}$ with this epitaxial structure and this emission wavelength. The effective front facet reflectivity of $R_{\mathrm{s}}=0.0015 \%$ is again the result of fitting eq. (2) to the measured data of the maximum frequency shift for the feedback power attenuation in the range of $\mu_{\mathrm{AT}}=-105 \mathrm{~dB}$ to $-75 \mathrm{~dB}$. This value is significantly below the front facet coating $R_{\mathrm{FF}}=0.3 \%$. However, if take into account the device internal coupling losses to the RW section based on a free propagating Gaussian beam in the tapered section and multiply this with the front facet reflectivity of $R_{\mathrm{FF}}=0.3 \%$ we get an effective front facet reflectivity of $R_{\mathrm{eff}}=0.0019 \%$. The simulated data based on the above-mentioned values matches the measured data for weak external feedback with feedback power attenuation below $\mu_{\mathrm{AT}} \approx-67 \mathrm{~dB}$. At this attenuation the simulated data has a step from 2 to 3 possible simultaneous modes as shown in Fig. $8 \mathrm{c}$ ). This step was not observed in the measurements. Only the simulated mode spacing between neighboring modes shown in Fig. 8 d) is in good agreement with the measured data over whole measured data range.
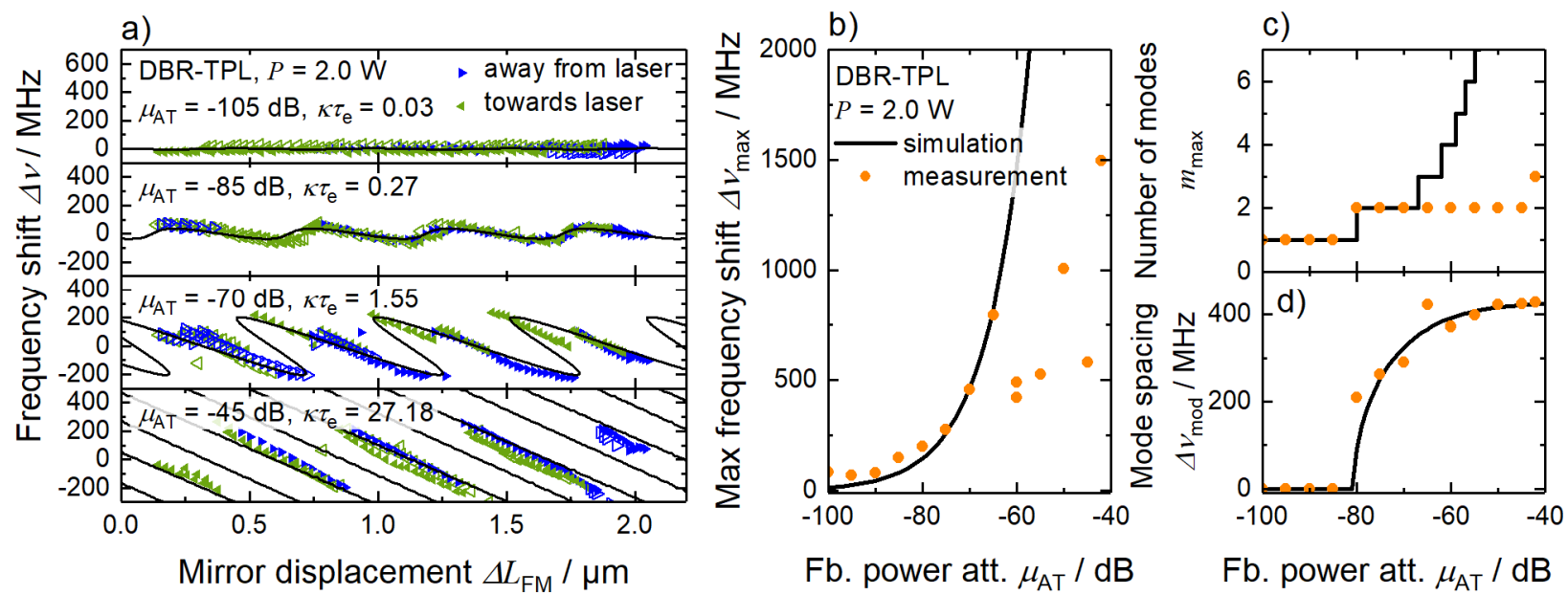

Fig. 8 a) Frequency shift over the mirror displacement for 4 different feedback power attenuations $\mu_{\mathrm{AT}}$ for the DBR tapered diode laser. The mirror movement direction is shown by the color of the symbols (green: towards laser diode, blue: away from laser diode). b) The maximum frequency shift $\Delta v_{\max }$, c) the maximum number of simultaneous possible modes and d) the mode spacing between neighboring modes are plotted over the feedback power attenuation $\mu_{\text {Ат }}$. The measured values (symbols) are compared for all graphs with simulation (solid black line).

\section{CONCLUSION}

We presented the results of an investigation on the influence of controlled external feedback on low and high power diode lasers with a frequency selective element. A DFB laser at $P=140 \mathrm{~mW}$ and $\lambda=975 \mathrm{~nm}$ was used as a reference laser to test the measurement method and the feedback setup. The measured data for the induced frequency shift are in excellent agreement with the simulated data based on the Van der Pol equation with an additional source term (eq. (1)). 
A DBR tapered diode laser at $P=2 \mathrm{~W}$ and $\lambda=1066 \mathrm{~nm}$ was used as an example of a high power diode laser with a frequency selective element. The measured data for weak external feedback is in good agreement with the simulated data. But for higher feedback power only the mode spacing between neighboring modes matched the prediction from the simulations.

The work is first step towards a better understanding of the influence of external feedback on high power diode lasers and could lead to high power diode lasers which are less prone to external feedback.

\section{ACKNOWLEDGMENT}

This work was supported by the European commission within the project MIB 667933-2, FBI 721766 and the Innovation Fund Denmark.

\section{REFERENCES}

[1] Tkach, R. W. and Chraplyvy, A. R., "Regimes of feedback effects in 1.5- $\mu$ m distributed feedback lasers," J. Light. Technol. 4(11), 1655-1661 (1986).

[2] Tromborg, B., Osmundsen, J. and Olesen, H., "Stability analysis for a semiconductor laser in an external cavity," IEEE J. Quantum Electron. 20(9), 1023-1032 (1984).

[3] Lang, R. and Kobayashi, K., "External optical feedback effects on semiconductor injection laser properties," IEEE J. Quantum Electron. 16(3), 347-355 (1980).

[4] Wenzel, H., Fricke, J., Klehr, A., Knauer, A. and Erbert, G., "High-power 980-nm DFB RW lasers with a narrow vertical far field," IEEE Photonics Technol. Lett. 18(6), 737-739 (2006).

[5] Müller, A., Zink, C., Fricke, J., Bugge, F., Erbert, G., Sumpf, B. and Tränkle, G., "Efficient, High Brightness $1030 \mathrm{~nm}$ DBR Tapered Diode Lasers With Optimized Lateral Layout,” IEEE J. Sel. Top. Quantum Electron. 23(6), 1-7 (2017).

[6] Batrak, D. V., Bogatova, S. A., Borodaenko, A. V., Drakin, A. E. and Bogatov, A. P., "Simulation of the material gain in quantum-well InGaAs layers used in 1.06- $\mu$ m heterolasers," Quantum Electron. 35(4), 316-322 (2005). 